\title{
Minimally invasive liver surgery: an update
}

\author{
Fulvio Calise $^{1} \cdot$ Luca Aldrighetti $^{2}$
}

Published online: 29 July 2015

(C) Italian Society of Surgery (SIC) 2015

It is a great pleasure for us to introduce this special issue of Updates in Surgery focused on "Minimally Invasive Surgery of the Liver" (MILS).

Indeed, this area has recently developed and grown thanks both to advances in new technologies and to efforts done by a new generation of surgeons: in 2015, minimally invasive liver surgery has proved its safety and efficacy when performed by teams with good experience in both laparoscopic and hepatic surgery, allowing a significant reduction of intraoperative blood loss, need for portal clamping, postoperative morbidity and shortening postoperative hospitalization, as a result of a more rapid functional recovery. Initial skepticisms regarding technical challenges and oncological adequacy have been overcome, and great enthusiasm has been raised in this newborn field. In 2014, the 2nd consensus conference on MILS was held in Morioka, Japan, and recommendations based on the strength of scientific evidences have been provided. Many training opportunities are now available for surgeons who want to get specific experience. Overall, the future of MILS seems to be promising.

Despite this, many topics still deserve in-depth study: this monographic volume tries to reply to many open issues and to certify and codify what has already been acknowledged in clinical practice; we are witnesses of a dramatic change in the way of approaching many liver diseases and

Luca Aldrighetti

aldrighetti.luca@hsr.it

1 Unit of Hepatobiliary Surgery and Liver Transplant Center, "Cardarelli" Hospital, Naples, Italy

2 Hepatobiliary Surgery Division, IRCCS San Raffaele Hospital, Milan, Italy have the duty of offer the best available treatment to our patients.

We have the honor of including an Editorial from Professor Wakabayashi who describes current trends of laparoscopic liver surgery and its evolution from Louisville to Morioka. The volume is then structured into two sessions, thanks to the contribution of Italian and international authors: in the first one, four review articles are provided, focusing on hot topics like HCC surgery, definition of segmentectomies, robotic approach and difficulty scores; in the second part, by means of original articles, contributions are provided to scientific knowledge on issues that are still debated, such as indications and contraindications of major resections, resections of posterosuperior segments, outcomes of metastases resection, MILS in high- and low-volume Italian centers, advantages and limitations of robotic approach, learning curve and accuracy of laparoscopic ultrasound.

We therefore wish to thank all the authors for their effort in providing a better insight into the present and future time of this revolutionary approach to liver surgery.

We do hope that this editorial effort may help Italian and foreign colleagues willing to develop their activity in minimally invasive MILS according to the principles of scientific knowledge and evidence-based medicine.

Luca Aldrighetti

Fulvio Calise 


\section{Compliance with ethical standards}

Conflict of interest The authors declare that they don't have any conflict of interest.

Ethical standard All procedures in our paper were in accordance with the ethical standards of the institutional as well as national research committee and with the 1964 Helsinki declaration and its later amendments.
Research involving human participants and/or animals This articles does not contain any studies with human participants or animal performed by any of the authors.

Informed consent No informed consent is required. 